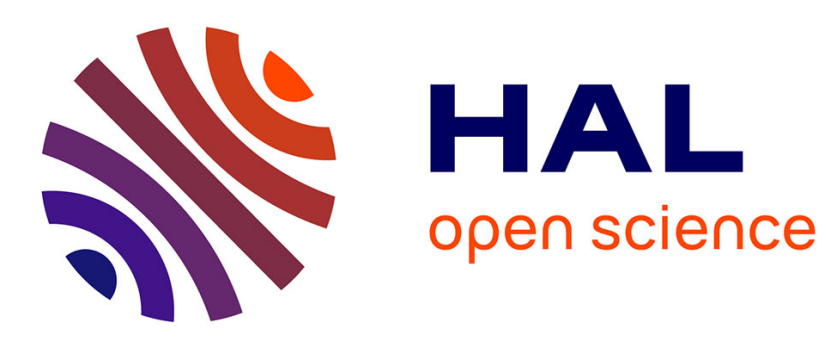

\title{
SABR TYPE STOCHASTIC VOLATILITY OPERATOR IN HILBERT SPACE
}

Raphaël Douady, Zeyu Cao

\section{To cite this version:}

Raphaël Douady, Zeyu Cao. SABR TYPE STOCHASTIC VOLATILITY OPERATOR IN HILBERT SPACE. 2020. hal-03018478

\section{HAL Id: hal-03018478 \\ https://hal-paris1.archives-ouvertes.fr/hal-03018478}

Preprint submitted on 22 Nov 2020

HAL is a multi-disciplinary open access archive for the deposit and dissemination of scientific research documents, whether they are published or not. The documents may come from teaching and research institutions in France or abroad, or from public or private research centers.
L'archive ouverte pluridisciplinaire HAL, est destinée au dépôt et à la diffusion de documents scientifiques de niveau recherche, publiés ou non, émanant des établissements d'enseignement et de recherche français ou étrangers, des laboratoires publics ou privés. 


\title{
SABR TYPE STOCHASTIC VOLATILITY OPERATOR IN HILBERT SPACE
}

\author{
RAPHAEL DOUADY ZEYU CAO
}

\begin{abstract}
Aвstract. In this paper, we define stochastic volatility operators in Hilbert space which are analogs to the widely-used SABR model [14] in finite dimensional case. We show the existence of the mild solution and some related regularity properties. Our proof is based on Leray-Schauder fixed point theorem and some priori inequalities on the stochastic operator processes we construct.
\end{abstract}

\section{INTRODUCTION}

It has been noticed for a long time that many financial problems could be naturally modeled with values in Banach spaces. Such ideas can be dated back to Douady [9], Musiela [16], etc. They are the pioneers who apply stochastic differential equations valued in Hilbert spaces to model the dynamics of evolution of instantaneous forward interest rate curves. Then Filipovic and his colleagues largely developed such methods, extended the classical Heath-Jarrow-Merton model and studied many further related geometric properties, see [10] [11] [12]. However, these methods mainly follow the no-arbitrage principle, and in the meantime, Cont, Bouchaud and etc [3] [18] suggested to model the forward interest rate curve as a vibrating string and added $D(x) \frac{\partial^{2}}{\partial x^{2}}$ as a regularizing term into their model. They argued that, although the model was not arbitrage-free, arbitrage opportunities were, in practice, eliminated by transaction costs. Such type of models gained increasing attention in recent years, in particular, after the 2008 financial crisis. One advantage of such an approach is that the regularity provided by this term avoids many issues of Filipovic's model, such as the wild behavior of the forward interest rate curve in the long run. A good comprehensive book which gives a nice overall introduction on this area is Carmona and Tehranchi [2]. Another way to model the evolution of interest rate curves is to use random field theory. D. P. Kennedy [15] is among the first to adopt such ideas. In a short remark at the end of his article, he also noticed and discussed the importance of the second order differential operator as a regularity term. An important and classic topic in interest rate modeling is stochastic volatility. Interest rate and volatility derivatives are very sensitive to the volatility of volatility, and many models on this aspect are introduced. SABR (stochastic alpha beta rho) model, Hagan and etc [14], is among the most popular and widely used and one of its key is that the volatility depends on some fractional power of the level of the forward rate. Such phenomena is also supported empirically, and its importance is discussed by Douady [8].

In this paper, we raise the question: how to construct infinite dimension version of stochastic volatility which is supposed to be a random process valued in some Hilbert space of operators. There is little work on such topics until recently Benth and Simonsen [1] introduce an infinite dimensional version of Heston stochastic volatility operator model. However, the volatility in their model is independent of the level of the forward rate curve. In order to handle SABR-type volatility operators, we develop two versions of models to relate volatility with level. One is a direct generalization of

Date: July 5, 2020.

Key words and phrases. Wiener Process in Hilbert Space, Stochastic Volatility, Operator Theory, Financial Modeling. 
the classic SABR model while the other one is more general involving more non-linearity. Along with these volatility operator models, we include the regular term $D(x) \frac{\partial^{2}}{\partial x^{2}}$. And under some technical conditions, we show the existence of mild solutions to our models.

Another reason why we should investigate infinite dimensional models is that, similar to studying on fluid as a PDE on state functions and a dynamical system modeling each molecule inside in physics, it is usually better to consider a large set of assets as an infinite dimensional family first, then study it as a continuous mathematical object, that will be discretised for numerical purposes. Also, when performing any kind of calibration, one should always check its infinite dimensional limit. Indeed, there is a strong relationship between the stability of the numerical method in finite dimensions and the solvability in the infinite dimensional limit. Such views are discussed in more details by Douady [7].

The paper is organized as follows: in section 2, basic notations and mathematical results are introduced, we also give the construction of the stochastic volatility operator models and necessary conditions, then in section 3, we state and provide the detailed proof of the existence of mild solution to our model. Then we make our assumptions more realistic and natural by considering an OrnsteinUhlenbeck process in section 4; in section 5, we generalize some regularity theorems with stochastic volatility operators; and we conclude this paper in section 6 with further remarks and plans for future work.

\section{Preliminaries and Model Construction}

In this section, we will introduce some necessary notations and basic results, then we will give the construction of our models.

2.1. Notations and Basic Settings. Now let's construct our model for instantaneous forward interest rate $v_{t}$ with stochastic volatility operator $\Sigma_{t}$. Let $\mathbb{H}$ denote the real Hilbert space $L^{2}(0, M)$ where $M$ denotes the time to maturity of interest rates. We also fix some complete probability space $\left(\Omega, \mathcal{F}, \mathcal{F}_{t}, \mathbb{P}\right)$, over which two Wiener processes are defined: a $Q$-Wiener process $W_{t}$ and a $R$-Wiener process $B_{t}$; both $Q$ and $R$ are covariance operators with finite trace. We also assume that there are two abstract real Hilbert spaces $\mathbb{U}$ and $\mathbb{V}$, where $W_{t}$ is $\mathbb{U}$-valued and $B_{t}$ is $\mathbb{V}$-valued. Without loss of generality, we may assume that the filtration $\mathcal{F}_{t}$ is generated by $W_{t}$ and $B_{t}$. For $\mathbb{U}($ resp. $\mathbb{V})$, let $\mathbb{U}_{Q}$ (resp. $\mathbb{V}_{R}$ ) denote a Hilbert subspace of $\mathbb{U}($ resp. $\mathbb{V})$ whose norm $(\cdot, \cdot)_{Q}\left(\operatorname{resp} .(\cdot, \cdot)_{R}\right)$ is defined as $(g, h)_{Q}=\left(g, Q^{-1} h\right)_{\mathbb{U}}$ (resp. $\left.(g, h)_{R}=\left(g, R^{-1} h\right)_{\mathbb{V}}\right)$. Also, let $\mathbb{L}_{2}\left(\mathbb{U}_{Q}, \mathbb{H}\right), \mathbb{L}_{2}\left(\mathbb{U}_{Q}, \mathbb{R}\right)$ and so forth denote the spaces of HilbertSchmidt Operator (HS-) between the two corresponding Hilbert spaces.

Now consider the unbounded operator

$$
A=D(x) \frac{\partial^{2}}{\partial x^{2}}+\frac{\partial}{\partial x}
$$

defined over $\mathbb{H}$ whose domain $\mathcal{D}(A)$ is $H^{2}(0, M) \cap H_{0}^{1}(0, M)$, where $H^{2}(0, M)$ denotes the Sobolev space over the open interval $(0, M)$, consisting of all the $L^{2}$ - functions having second order derivative in $L^{2}$ and $H_{0}^{1}(0, M)$ denotes those in $H^{2}(0, M)$ with compact support. The function $D(x)$ is assumed strictly positive. 
Let us now consider $\Delta_{t}$ a continuous deterministic process of mappings from $\mathbb{H}$ to itself representing the drift of the yield curve process. We assume that for a.e. $(t, \omega) \in[0, T] \times \Omega, \Delta_{t}(\omega, \cdot)$ is $\mathcal{F}_{t}$-adapted and satisfies the integral condition (which we call Condition $C$ in section 3.3.4): there exist two non-negative continuous functions $f_{1}$ and $f_{2}$ defined over $[0, T]$ such that

$$
\text { for any } t \in[0, T] \text { and any } h(x) \in \mathbb{H}, E\left\|\Delta_{t}(h(x))\right\|^{2} \leq f_{1}(t) E\|h(x)\|^{2}+f_{2}(t) .
$$

Then the model for the instantaneous forward rates is constructed as:

$$
d v_{t}=A v_{t} d t+\Delta_{t}\left(v_{t}\right) d t+\Sigma_{t}\left(v_{t}\right) d W_{t}, t \in[0, T], v_{0} \in \mathbb{H},
$$

where $\Sigma_{t}$ is the stochastic volatility operator process so that $\Sigma_{t}\left(v_{t}\right)$ is valued in the real Hilbert space $\mathbb{L}_{2}\left(\mathbb{U}_{Q}, \mathbb{H}\right)$. The construction of it relies on another stochastic process $\sigma_{t}$ valued in some abstract Hilbert space $\widetilde{\mathbb{H}}$ which will be specified below. Before that let's first look at some notations and classic results regarding the existence of $\sigma_{t}$.

Summary Our construction is: the yield curves are in space $\mathbb{H}$, driven by random noise $\mathbb{W}_{t} \in \mathbb{U}_{Q}$. The volatility operator process $\Sigma_{t} \in \mathbb{L}_{2}\left(\mathbb{U}_{Q}, \mathbb{H}\right)$, and it's a function of another more elementary process $\sigma_{t}, \Sigma_{t}=F\left(\sigma_{t}\right)$, where $\sigma_{t} \in \widetilde{\mathbb{H}}$. Process $\sigma_{t}$ is driven by noise $\mathbb{B}_{t} \in \mathbb{V}_{R}$. The volatility operator of volatility operator $\Sigma_{t}$, denoted as $G\left(t, \sigma_{t}\right)$, is valued in $\mathbb{L}_{2}\left(\mathbb{V}_{R}, \widetilde{\mathbb{H}}\right)$. The exact forms of $\widetilde{\mathbb{H}}$ and function $F$ will be specified in Model 1 and Model 2 introduced below.

2.2. Classic Results on Existence of Solutions for SDEs valued in Hilbert spaces. Now we can consider the initial value problem of stochastic evolution equations for the process $\sigma_{t}$ in Hilbert spaces:

$$
\left\{\begin{array}{l}
d \sigma_{t}=\widetilde{A} \sigma_{t}+F\left(t, \sigma_{t}\right) \sigma_{t}+G\left(t, \sigma_{t}\right) d B_{t}, \quad t \in(0, T) \\
\sigma_{0}=h \in \widetilde{\mathbb{H}}
\end{array}\right.
$$

where $\widetilde{A}$ is a linear operator (possibly unbounded) defined on $\widetilde{\mathbb{H}}$ whose domain $\mathcal{D}(\widetilde{A})$ is dense in $\widetilde{\mathbb{H}}$ and $\widetilde{A}$ generates a strongly continuous $C_{0}$-semigroup $\Gamma_{t}$. Moreover, for a.e. $(t, \omega) \in[0, T] \times \Omega$, $F(t, \omega): \widetilde{\mathbb{H}} \rightarrow \widetilde{\mathbb{H}}$ and $G(t, \omega): \widetilde{\mathbb{H}} \rightarrow \mathbb{L}_{2}\left(\mathbb{V}_{R}, \widetilde{\mathbb{H}}\right)$ are $\mathcal{F}_{t}$-adapted and predictable.

Usually the above problem does not have a strong solution (i.e. an actual solution lying in $\widetilde{\mathbb{H}}$ ), instead, people consider weak and mild solutions. Let's recall the definitions of these three types of solutions and their relations below.

Strong solution:

Consider an $\mathcal{F}_{t}$-adapted stochastic process $\sigma_{t}$ satisfying:

$$
\left.\mathbb{P}\left(\int_{0}^{T}\left\|\widetilde{A} \sigma_{t}\right\|_{\widetilde{\mathbb{H}}}\right) d t<\infty\right)=1 \text { and } \mathbb{P}\left(\int_{0}^{T}\left(\left\|F\left(t, \sigma_{t}\right)\right\|+\left\|G\left(t, \sigma_{t}\right)\right\|^{2}\right) d t<\infty\right)=1
$$

$\sigma_{t}$ is a continuous process valued in $\widetilde{\mathbb{H}}$ and $\sigma_{t}$ is in $\mathcal{D}(\widetilde{A})$ almost everywhere. 
Then $\sigma_{t}$ is a strong solution if for any $t \leq T$, almost surely,

$$
\sigma_{t}=h+\int_{0}^{t} \widetilde{A} \sigma_{s}+F\left(s, \sigma_{s}\right) d s+\int_{0}^{t} G\left(s, \sigma_{s}\right) d B_{s}
$$

Weak solution:

Consider an $\mathcal{F}_{t}$-adapted stochastic process $\sigma_{t}$ satisfying:

$$
\left.\mathbb{P}\left(\int_{0}^{T}\left\|\sigma_{t}\right\|_{\mathbb{\mathbb { H }}}\right) d t<\infty\right)=1 \text { and } \mathbb{P}\left(\int_{0}^{T}\left(\left\|F\left(t, \sigma_{t}\right)\right\|+\left\|G\left(t, \sigma_{t}\right)\right\|^{2}\right) d t<\infty\right)=1 .
$$

Then $\sigma_{t}$ is a weak solution if for any $h \in \mathcal{D}\left(\widetilde{A^{*}}\right)$ and $t \leq T, \mathbb{P}$ - almost surely:

$$
\left\langle\sigma_{t}, h\right\rangle=\left\langle\sigma_{0}, h\right\rangle+\int_{0}^{t}\left(\left\langle\sigma_{s}, \widetilde{A}^{*} h\right\rangle+\left\langle F\left(s, \sigma_{s}\right), h\right\rangle\right) d s+\int_{0}^{t}\left\langle h, G\left(s, \sigma_{s}\right) d B_{s}\right\rangle,
$$

where $\widetilde{A}^{*}$ denotes the dual map, and $\langle>$ denotes the inner product of Hilbert space $\widetilde{\mathbb{H}}$.

Mild solution:

Consider an $\mathcal{F}_{t}$-adapted stochastic process $\sigma_{t}$ satisfying:

$$
\left.\mathbb{P}\left(\int_{0}^{T}\left\|\sigma_{t}\right\|_{\widetilde{\mathbb{H}}}\right) d t<\infty\right)=1 \text { and } \mathbb{P}\left(\int_{0}^{T}\left(\left\|F\left(t, \sigma_{t}\right)\right\|+\left\|G\left(t, \sigma_{t}\right)\right\|^{2}\right) d t<\infty\right)=1 .
$$

The stochastic process $\sigma_{t}$ is a mild solution if:

$$
\sigma_{t}=\Gamma_{t} h+\int_{0}^{t} \Gamma_{t-s} F\left(s, \sigma_{s}\right) d s+\int_{0}^{t} \Gamma_{t-s} G\left(s, \sigma_{s}\right) d B_{s}
$$

In particular, a strong solution is a weak solution, and one can prove that a weak solution is a mild solution but not necessary conversely. The existence of strong solutions require very binding assumptions. However, if $E\left(\int_{0}^{T}\left\|G\left(t, \sigma_{t}\right)\right\|^{2} d t\right)<\infty$, a mild solution is also a weak solution (see Theorem 3.2 [13]), which is very useful for the future numerical implementation purpose. In practice, it is usually easier to prove the existence of mild solutions. For this reason, we first develop the existence theory of mild solutions in this paper.

We now present some classic results on the existence of solutions and some related properties which will be used later. 
Theorem 2.1. (Theorem 6.5 [5]) For the above stochastic evolution equation problem, assume that:

1. For any $h \in \widetilde{\mathbb{H}}, t \in[0, T], F(t, h, \omega)$ is a locally integrable $\widetilde{\mathbb{H}}$-valued process, and $G(t, h, \omega)$ is predictable.

2. For some $p \geq 2$, there exist positive constants $a_{1}$ and $a_{2}$ such that

$$
E \int_{0}^{T}\left[\|F(s, 0, \omega)\|^{p}+\|G(s, 0, \omega)\|_{R}^{p}\right] d s \leq a_{1},
$$

and for any $g \in \widetilde{\mathbb{H}}$,

$$
\|F(t, g, \omega)-F(t, 0, \omega)\|^{2}+\|G(t, g, \omega)-G(t, 0, \omega)\|_{R}^{2} \leq a_{2}\left(1+\|g\|^{2}\right),
$$

for a.e. $(t, \omega) \in[0, T] \times \Omega$.

3. There exist a constant $c>0$ such that, for any $g, h \in \widetilde{\mathbb{H}}$,

$$
\|F(t, g, \omega)-F(t, h, \omega)\|+\|G(t, g, \omega)-G(t, h, \omega)\|_{R} \leq c\|g-h\|,
$$

for a.e. $(t, \omega) \in[0, T] \times \Omega$.

Then, for $h \in \widetilde{\mathbb{H}}$ and $p \geq 2$, the above initial value stochastic evolution equation problem has a unique mild solution $\sigma_{t}, 0 \leq t \leq T$ with $\sigma_{t} \in C\left([0, T] ; L^{p}(\Omega, \widetilde{\mathbb{H}})\right)$ such that

$$
E\left\{\sup _{0 \leq t \leq T}\left\|u_{t}\right\|^{p}\right\} \leq K_{p}(T)\left\{1+E \int_{0}^{T}\left[\|F(s, 0, \omega)\|^{p}+\|G(s, 0, \omega)\|_{R}^{p}\right] d s\right\},
$$

for some constant $K_{p}(T)>0$. For $p>2$, the solution has continuous sample paths.

Lemma 2.1. (Theorem 4.37 [6]) Assume that $p \geq 2$ and let $\Phi$ be an $\mathbb{L}_{2}\left(\mathbb{U}_{Q}, \mathbb{H}\right)$-valued predictable process such that $E \int_{0}^{T}\|\Phi(s)\|^{p} d s<\infty$, then

$$
E \sup _{0 \leq t \leq T}\left|\int_{0}^{t} \Phi(s) d W_{s}\right|^{p} \leq c_{p}\left[\int_{0}^{T}\left(E\|\Phi(s)\|_{Q}^{p}\right)^{\frac{2}{p}} d s\right]^{\frac{p}{2}},
$$

where $c_{p}$ is some positive constant. Moreover, if for any $t, t \mapsto \int_{0}^{t} \Phi(s) d W_{s}$ is continuous, then $\sup _{0 \leq t \leq T}\left\|\int_{0}^{t} \Phi(s) d W_{s}\right\|^{p}$ is measurable, and we can exchange sup and expectation.

2.3. Construction of Volatility Operator Process. First, apply Theorem $\mathbf{2 . 1}$ with $p \geq 4$ to construct the process $\sigma_{t}$. Then we can claim that $\sigma_{t}$ has continuous sample path and there exists a positive constant $\widetilde{C}$ only depending on $T$ such that for any $t \in[0, T], E\left\|\sigma_{t}\right\|^{p} \leq \widetilde{C}$. Now, we propose two different kind of constructions of stochastic volatility operator models $\Sigma_{t}$ using $\sigma_{t}$.

Model 1 (Infinite Dimension SABR) :

$\widetilde{\mathbb{H}}=\mathbb{L}_{2}\left(\mathbb{U}_{Q}, \mathbb{R}\right)$, where $\mathbb{R}$ denotes the space of real numbers. $p=4$. The stochastic volatility operator $\Sigma_{t}$ is defined as

$$
\Sigma_{t}(h)=|h|^{\beta} \sigma_{t},
$$


for any $\mathrm{h} \in \mathbb{H}$, where $|\cdot|$ denotes the standard absolute value, $0<\beta<0.5$.

It is not hard to see that for any element $h \in \mathbb{H}, \Sigma_{t}(h) \in \mathbb{L}_{2}\left(\mathbb{U}_{Q}, \mathbb{H}\right)$. Indeed,

$$
\left\|\Sigma_{t}(h)\right\|_{\mathbb{L}_{2}\left(\mathbb{U}_{Q}, \mathbb{H}\right)}^{2}=\|h\|_{\mathbb{H}^{\prime}}^{2}\left\|\sigma_{t}\right\|_{\mathbb{L}_{2}\left(\mathbb{U}_{Q}, \mathbb{R}\right)}^{2},
$$

and also notice that if $h \in \mathbb{H}$, then so is $|h|^{\beta}$.

Model 2 ( A general nonlinear volatility operator model):

First, let's embed $\mathbb{H}$ into $\mathbb{L}_{2}\left(\mathbb{H}_{Q}, \mathbb{H}\right)$ : choose some unitary element $e$ in $\mathbb{H}_{Q}$, consider

$$
\begin{aligned}
\iota: \mathbb{H} & \rightarrow \mathbb{L}_{2}\left(\mathbb{H}_{Q}, \mathbb{H}\right) \\
h & \mapsto h \otimes<e, \cdot>,
\end{aligned}
$$

this is an isometric embedding,thus, $\mathbb{H}$ can be regarded as a subspace of $\mathbb{L}_{2}\left(\mathbb{H}_{Q}, \mathbb{H}\right)$.

Now assume that $P(x)$ is a polynomial of degree $d$ of one variable, and let $\mathbb{U}$ be $\mathbb{L}_{2}\left(\mathbb{H}_{Q}, \mathbb{H}\right)$, and $\widetilde{\mathbb{H}}$ be $\mathbb{L}_{2}\left(\mathbb{L}_{2}\left(\mathbb{H}_{Q}, \mathbb{H}\right), \mathbb{L}_{2}\left(\mathbb{H}_{Q}, \mathbb{H}\right)\right.$ ), then by Theorem 2.1 (with $4 d \leq p$ ) we get the $\widetilde{\mathbb{H}}$-valued process $\sigma_{t}$, and the stochastic volatility operator $\Sigma_{t}(\cdot)$ is defined as the restriction of $P\left(\sigma_{t}(|\cdot| \beta)\right)$ on $\mathbb{H}, 0<\beta<0.5$.

The whole construction can be summarised by the following diagrams:

$$
\begin{aligned}
\sigma_{t}(|\cdot| \beta): \mathbb{L}_{2}\left(\mathbb{H}_{Q}, \mathbb{H}\right) & \rightarrow \mathbb{L}_{2}\left(\mathbb{L}_{2}\left(\mathbb{H}_{Q}, \mathbb{H}\right), \mathbb{L}_{2}\left(\mathbb{H}_{Q}, \mathbb{H}\right)\right) \\
v_{t} & \rightarrow \sigma_{t}\left(\left|v_{t}\right|^{\beta}\right)
\end{aligned}
$$

The commutative diagram for construction of $\Sigma_{t}$ :

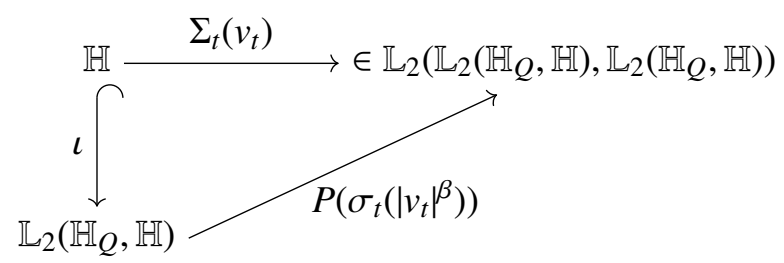

Remark: for any element $h \in \mathbb{H}$ and any element $\omega \in \Omega$, consider the norm and its upper bound of $\Sigma_{t}(\omega, h)$.

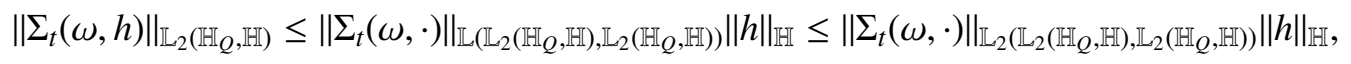

where $\mathbb{L}\left(\mathbb{H}_{Q}, \mathbb{H}\right)$ denotes the space of bounded linear operators between two real Hilbert spaces.

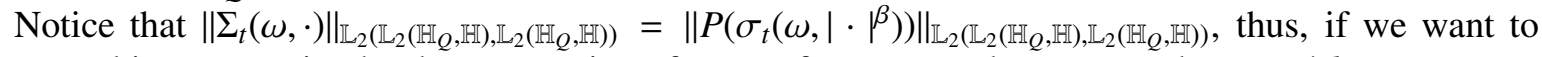
control its expectation by the expectation of norm of $\sigma_{t}$, we need to assume that $p \geq 4 d$. 


\section{Main Theorem and Its Proof}

Now we are ready to state our main theorem and its proof.

\subsection{Main Theorem.}

Theorem 3.1. Let's first recall our model and the assumptions, the evolution equation of yield curve $v_{t}$ is, defined as in (2.1),

$$
d v_{t}=A v_{t} d t+\Delta_{t}\left(v_{t}\right) d t+\Sigma_{t}\left(v_{t}\right) d W_{t}, t \in[0, T], v_{0} \in \mathbb{H},
$$

where $A=D(x) \frac{\partial^{2}}{\partial x^{2}}+\frac{\partial}{\partial x}$, an unbounded elliptic operator; $\Delta_{t}$ is the drift term satisfying Condition $C$; and the stochastic volatility operator process $\Sigma_{t}$ is a function of the elementary process $\sigma_{t}$ defined above in Model 1 and Model 2, where $\sigma_{t}$ satisfies

$$
\left\{\begin{array}{l}
d \sigma_{t}=\widetilde{A} \sigma_{t}+F\left(t, \sigma_{t}\right) \sigma_{t}+G\left(t, \sigma_{t}\right) d B_{t}, \quad t \in(0, T) \\
\sigma_{0}=h \in \widetilde{\mathbb{H}},
\end{array}\right.
$$

where $\widetilde{A}, F(t, \cdot)$ and $G(t, \cdot)$ satisfy the conditions in Theorem 2.1 and Lemma 2.1, then operator A generates a compact, analytic, strongly continuous semigroup, and Problem (2.1) has at least one mild solution.

Remark Indeed, Condition $C$ is very important to the existence of global mild solution, and we will show that by our construction of Model 1 and Model 2, the volatility operator process $\Sigma_{t}(\cdot)$ also satisfies Condition $C$.

\subsection{Proof of Main Theorem.}

3.2.1. Step 1. In this part, we prove that the operator A generates a compact, analytic, strongly continuous semigroup.

Let's follow the definitions in [17]. The principal part of $A$ is $D(x) \frac{\partial^{2}}{\partial x^{2}}$ and it is strongly elliptic of order $2 m(m=1)$ due to the assumption of $D(x)$.

Then by Gárding's inequality (Theorem 2.2 Chapter 7 [17]), there exist $c_{0}>0, \lambda_{0} \geq 0$ such that for any $u \in \mathcal{D}(A)$,

$$
\operatorname{Re}(-A u, u)_{0} \geq c_{0}\|u\|_{1,2}^{2}-\lambda_{0}\|u\|_{0,2}^{2} .
$$

By Theorem 2.3 Chapter 7 [17], for any $\lambda$ satisfying $\operatorname{Re} \lambda \geq \lambda_{0}$, and every $f \in \mathbb{H}$, there exists a unique $u \in \mathcal{D}(A)$ such that

$$
A u-\lambda u=f,
$$

which indicates that $A-\lambda \mathrm{Id}$ is full. Then by Theorem 2.7 Chapter 7 [17], $A$ generates an analytic semigroup $\left\{G_{t}\right\}_{t \geq 0}$.

Due to Theorem 4.29 Chapter II [10], to show $A$ generates an immediately compact semigroup (in other words, for any $t>0, G_{t}$ is compact), one only needs to show :

1. $\left\{G_{t}\right\}_{t \geq 0}$ is immediately norm continuous, in other words, $G_{t}$ is norm continuous for any $t>0$. 
2. A has compact resolvent.

Indeed, part 1 is true since $A$ generates an analytic semigroup. To show that $A$ has a compact resolvent, we need the following result (Exercise 4.30 (4) Chapter II [10]):

Let $X=L^{2}(\widetilde{\Omega}), \widetilde{\Omega}$ is a bounded interval in $R$. If $(A, \mathcal{D}(A))$ is an operator on $\mathrm{X}$ without empty resolvent set and $\mathcal{D}(A) \subset W^{1,2}(\widetilde{\Omega})$, then $A$ has compact resolvent.

In our case, $\widetilde{\Omega}$ is $(0, M)$, by notation and definition, $\mathcal{D}(A) \subset H_{0}^{1}(0, M)=W_{0}^{1,2}(0, M) \subset W^{1,2}(0, M)$, thus, we only need to show that $\rho(A) \neq \emptyset$.

However, by definition of resolvent set $\rho$ of a closed unbounded operator, we have $\rho(A) \neq \emptyset$ since for any $\lambda, \operatorname{Re} \lambda \geq \lambda_{0}, \lambda \mathrm{Id}-A$ is bijective between $\mathcal{D}(A)$ and $\mathbb{H}$. Notice that $A$ is closed since it generates strongly continuous semigroup, see Theorem 1.4 Chapter II [10].

\subsubsection{Step 2. Preparation}

The set of all strongly measurable, square-integrable $\mathbb{H}$-valued random variables, denoted by $L^{2}(\Omega, \mathbb{H})$, is a Hilbert space with norm $\|v(\cdot)\|_{L^{2}}=\left(E\left(\|v(\cdot, \omega)\|^{2}\right)\right)^{\frac{1}{2}}$. A subspace is $L_{0}^{2}(\Omega, \mathbb{H})=\{v \in$ $L^{2}(\Omega, \mathbb{H}): f$ is $\mathcal{F}_{0}$-measurable $\}$.

Let $I$ denotes some closed finite subinterval of $[0, \infty)$. Let $C\left(I, L^{2}(\Omega, \mathbb{H})\right)$ denote the space of all $\mathcal{F}_{t}$-adapted, measurable, and continuous (mean-squared continuous) processes from $I$ to $L^{2}(\Omega, \mathbb{H})$ satisfying $\sup _{t \in I} E\left(\|v(t)\|^{2}\right) \leq \infty$. It is easy to see that such a space is a Banach space with norm:

$$
\|v\|_{C}:=\left(\sup _{t \in I} E\left(\|v(t)\|^{2}\right)\right)^{\frac{1}{2}}
$$

Now, based on the definition of mild solution, let's define an operator $\Psi$ on $C\left(I, L^{2}(\Omega, \mathbb{H})\right)$, assuming $I=\left[t_{0}, t_{0}+\delta\right]$ where $\delta$ is some small positive real number:

$$
\Psi\left(v_{t}\right):=G_{t-t_{0}} v_{t_{0}}+\int_{t_{0}}^{t} G_{t-s} \Delta_{s}\left(v_{s}\right) d s+\int_{t_{0}}^{t} G_{t-s} \Sigma_{s}\left(v_{s}\right) d W_{s}, t \in I
$$

3.2.3. Step 3. In this part, we will show that the operator $\Psi$ is well-defined, in other word, its image is in $C\left(I, L^{2}(\Omega, \mathbb{H})\right)$, and it is a continuous map between Banach spaces.

1) $\Psi\left(v_{t}\right) \in C\left(I, L^{2}(\Omega, \mathbb{H})\right)$

$$
\begin{aligned}
& E\left(\left\|\Psi\left(v_{t}\right)\right\|^{2}\right) \leq E\left(\left(\left\|G_{t-t_{0}} v_{t_{0}}\right\|+\left\|\int_{t_{0}}^{t} G_{t-s} \Delta_{s}\left(v_{s}\right) d s\right\|+\left\|\int_{t_{0}}^{t} G_{t-s} \Sigma_{s}\left(v_{s}\right) d W_{s}\right\|\right)^{2}\right) \\
& \leq 3 E\left(\left\|G_{t-t_{0}} v_{t_{0}}\right\|^{2}\right)+3 E\left(\left\|\int_{t_{0}}^{t} G_{t-s} \Delta_{s}\left(v_{s}\right) d s\right\|^{2}\right)+3 E\left(\left\|\int_{t_{0}}^{t} G_{t-s} \Sigma_{s}\left(v_{s}\right) d W_{s}\right\|^{2}\right) .
\end{aligned}
$$


Let $M\left(t_{0}\right)$ be $\max _{t \in I}\left\|G_{t}\right\|$, then apply Lemma 2.1 with $p=2$,

$$
E\left\|\Psi\left(v_{t}\right)\right\|^{2} \leq 3 M\left(t_{0}\right)^{2}\left\|E v_{t_{0}}\right\|^{2}+3 M\left(t_{0}\right)^{2}\left\|E \int_{t_{0}}^{t} \Delta_{s}\left(v_{s}\right) d s\right\|^{2}+3 \hat{c} \cdot M\left(t_{0}\right)^{2} \int_{t_{0}}^{t} E\left\|\Sigma_{s}\left(v_{s}\right)\right\|^{2} d s,
$$

where $\hat{c}$ is some positive constant. By our assumptions, it is clear that the first two terms are finite. Let's show that this still holds true for the last term:

For Model 1:

$$
\int_{t_{0}}^{t} E\left\|\Sigma_{s}\left(v_{s}\right)\right\|^{2} d s=\int_{t_{0}}^{t} E\left(\left\|\sigma_{t}\right\|^{2}\left\|\left|v_{t}\right|^{\beta}\right\|^{2}\right) d s \leq \int_{t_{0}}^{t}\left(E\left\|\sigma_{s}\right\|^{4}\right)^{\frac{1}{2}}\left(E\left\|\left|v_{s}\right|^{\beta}\right\|^{4}\right)^{\frac{1}{2}} d s,
$$

notice that $\left(E\left\|\sigma_{s}\right\|^{4}\right)^{\frac{1}{2}}$ is bounded by our assumption of Model 1, and

$$
E\left\|\left|v_{s}\right|^{\beta}\right\|^{4}=E\left(\int_{0}^{M}\left|v_{s}(x)\right|^{2 \beta} d x\right)^{2} \leq M^{2-2 \beta}\left[E\left(\int_{0}^{M}\left|v_{s}(x)\right|^{2} d x\right)^{2 \beta}\right],
$$

notice that $E\left(\int_{0}^{M}\left|v_{s}(x)\right|^{2} d x\right)^{2 \beta} \lesssim\left[E\left(\int_{0}^{M}\left|v_{s}(x)\right|^{2} d x\right)\right]^{2 \beta}$, which is bounded for any $s$.

For Model 2:

$$
E\left\|\Sigma_{t}\left(v_{t}\right)\right\|^{2}=E\left\|P\left(\sigma_{t}\right)\left(\left|v_{t}\right|^{\beta}\right)\right\|^{2} \leq E\left\|P\left(\sigma_{t}\right)\right\|^{2}\left\|\left|v_{t}\right|^{\beta}\right\|^{2} \leq\left[E\left\|P\left(\sigma_{t}\right)\right\|^{4}\right]^{\frac{1}{2}}\left[E\left\|\left|v_{t}\right|^{\beta}\right\|^{4}\right]^{\frac{1}{2}},
$$

and we already know that $\left[E\left\|\left|v_{t}\right|^{\beta}\right\|^{4}\right]^{\frac{1}{2}}$ is bounded from the analysis above in Model 1 ; notice that $E\left\|P\left(\sigma_{t}\right)\right\|^{4} \lesssim E\left\|\sigma_{t}\right\|^{4 d}$ since $P(x)$ is a polynomial of degree $d$. By our assumption for Model 2, this is also finite.

Thus, in either case the last integral term is finite, and we conclude that for any fixed $t \in I$, $\Psi\left(v_{t}\right) \in L^{2}(\Omega, \mathbb{H})$.

Moreover, to show the process $\psi\left(v_{t}\right)$ is continuous from $I$ to $C\left(I, L^{2}(\Omega, \mathbb{H})\right)$, one only needs to show that as $s \rightarrow t, E\left\|\Psi\left(v_{s}\right)-\Psi\left(v_{t}\right)\right\|^{2} \rightarrow 0$, but this is easy to see from the estimations for the two models above.

A Remark on the Upper Bound of $\beta$ : We have assumed that $\beta<0.5$, indeed, we can see from the arguments below that we only need this restriction instead of $\beta<1$ to show that $E\left\|\Sigma_{t}\left(v_{t}\right)\right\|^{2}$ is finite in our two models above for a general $\sigma_{t}$, since we only assume that $\sigma_{t}$ has finite $4 t h$ (resp. (4d)th) moment for Model 1 (resp. Model 2). Now, if the process $\sigma_{t}$ has higher finite moments, or ideally, finite moments for any order, then we have the following arguments to increase the upper bound of $\beta$. Let's use Model 1 as an example to illustrate the idea, similar procedure works for Model 2.

Assume that $E\left\|\sigma_{t}\right\|^{4 n}<\infty$, where $n$ is some positive integer, then 


$$
\begin{array}{r}
\int_{t_{0}}^{t} E\left\|\Sigma_{s}\left(v_{s}\right)\right\|^{2} d s=\int_{t_{0}}^{t} E\left(\left\|\sigma_{t}\right\|^{2}\left\|\left|v_{t}\right|^{\beta}\right\|^{2}\right) d s \leq \int_{t_{0}}^{t}\left(E\left\|\sigma_{s}\right\|^{4 n}\right)^{\frac{1}{2 n}}\left(E\left\|\left|v_{s}\right|^{\beta}\right\|^{\frac{4 n}{2 n-1}}\right)^{\frac{2 n-1}{2 n}} d s \\
\lesssim \int_{t_{0}}^{t}\left(E\left\|\left|v_{s}\right|^{\beta}\right\|^{\frac{4 n}{2 n-1}}\right)^{\frac{2 n-1}{2 n}} d s,
\end{array}
$$

thus only need to show $E\left\|\left|v_{s}\right|^{\beta}\right\|^{\frac{4 n}{2 n-1}}$ is bounded, in fact:

$$
E\left\|\left|v_{s}\right|^{\beta}\right\|^{\frac{4 n}{2 n-1}}=E\left(\int_{0}^{M}\left|v_{s}(x)\right|^{2 \beta} d x\right)^{\frac{2 n}{2 n-1}} \lesssim E\left(\int_{0}^{M}\left|v_{s}(x)\right|^{2} d x\right)^{\frac{2 n \beta}{2 n-1}},
$$

as long as $\frac{2 n \beta}{2 n-1}<1$, we can apply Hölder's inequality to get the desired finiteness estimation:

$$
E\left(\int_{0}^{M}\left|v_{s}(x)\right|^{2} d x\right)^{\frac{2 n \beta}{2 n-1}} \lesssim\left[E\left(\int_{0}^{M}\left|v_{s}(x)\right|^{2} d x\right)\right]^{\frac{2 n \beta}{2 n-1}},
$$

in other words, the upper bound of $\beta$ is $\frac{2 n-1}{2 n}$, which can be as close to 1 as desired with $n$ large enough.

Such process $\sigma_{t}$ with finite moments of high orders does exist and is indeed very natural, for instance, if $\sigma_{t}$ is either Gaussian or CIR type, then we have the necessary finite moments conditions. In particular, the Ornstein-Uhlenbeck Process, which is considered in section 4, is a good example.

2) Let's show that $\Psi: C\left(I, L^{2}(\Omega, \mathbb{H})\right) \rightarrow C\left(I, L^{2}(\Omega, \mathbb{H})\right)$ is a continuous map between Banach spaces.

Clearly, we indeed only need to show that $v_{t} \mapsto \int_{t_{0}}^{t} G_{t-s} v_{s} d W_{s}$ is a continuous map.

Assume that $v_{t}^{n}$ converges to $v_{t}$ as $n \rightarrow \infty$ in $C\left(I, L^{2}(\Omega, \mathbb{H})\right.$, in other words, $\sup _{t \in I} E\left\|v_{t}^{n}-v_{t}\right\|^{2}$ converges to 0 , as $n \rightarrow \infty$. Then for any $n$, let's estimate:

$$
\int_{t_{0}}^{t} G_{t-s} \Sigma_{s}\left(v_{s}^{n}\right) d W_{s}-\int_{t_{0}}^{t} G_{t-s} \Sigma_{s}\left(v_{s}\right) d W_{s}=\int_{t_{0}}^{t} G_{t-s} \Sigma_{s}\left(v_{s}^{n}-v_{s}\right) d W_{s}
$$

For Model 1:

$$
\begin{aligned}
E\left\|\int_{t_{0}}^{t} G_{t-s} \Sigma_{s}\left(v_{s}^{n}-v_{s}\right) d W_{s}\right\|^{2} & =E\left\|\int_{t_{0}}^{t} G_{t-s} \sigma_{s}\left(\left.\left|v_{s}^{n} \beta-\right| v_{s}\right|^{\beta}\right) d W_{s}\right\|^{2} \\
& \lesssim M\left(t_{0}\right)^{2} \int_{t_{0}}^{t} E\left\|\left(\left|v_{s}^{n}\right|^{\beta}-\left|v_{s}\right|^{\beta}\right) \cdot \sigma_{s}\right\|^{2} d s,
\end{aligned}
$$

due to Lemma 2.1.

Then

$$
E\left\|\int_{t_{0}}^{t} G_{t-s} \Sigma_{s}\left(v_{s}^{n}-v_{s}\right) d W_{s}\right\|^{2} \lesssim M\left(t_{0}\right)^{2} \int_{t_{0}}^{t}\left\{E\left\|\sigma_{s}\right\|^{4}\right\}^{\frac{1}{2}} \cdot\left\{E\left\|\left|v_{s}^{n}\right|^{\beta}-\left|v_{s}\right|^{\beta}\right\|^{4}\right\}^{\frac{1}{2}} d s,
$$


by assumption $E\left\|\sigma_{s}\right\|^{4}$ is uniformly bounded over $I$, thus,

$$
E \|\left.\int_{t_{0}}^{t} G_{t-s} \Sigma_{s}\left(v_{s}^{n}-v_{s}\right) d W_{s}\right|^{2} \lesssim \int_{t_{0}}^{t}\left\{E\left\|\left|v_{s}^{n}\right|^{\beta}-\left|v_{s}\right|^{\beta}\right\|^{4}\right\}^{\frac{1}{2}} d s .
$$

For Model 2: apply Lemma 2.1,

$$
\begin{aligned}
E\left\|\int_{t_{0}}^{t} G_{t-s} \Sigma_{s}\left(v_{s}^{n}-v_{s}\right) d W_{s}\right\|^{2} & =E \|\left.\int_{t_{0}}^{t} G_{t-s} P\left(\sigma_{s}\right)\left(\left|v_{s}^{n}\right|^{\beta}-\left|v_{s}\right|^{\beta}\right) d W_{s}\right|^{2} \\
& \lesssim M\left(t_{0}\right)^{2} \int_{t_{0}}^{t} E\left\|P\left(\sigma_{s}\right)\left(\left|v_{s}^{n}\right|^{\beta}-\left|v_{s}\right|^{\beta}\right)\right\|^{2} d s .
\end{aligned}
$$

Notice that $\left\|P\left(\sigma_{t}\right)\right\|_{\mathbb{L}} \leq\left\|P\left(\sigma_{t}\right)\right\|_{\mathbb{L}_{2}}$, where $\|\cdot\|_{\mathbb{L}}$ denotes the norm of bounded operator and $\|\cdot\|_{\mathbb{L}_{2}}$ denotes the norm of Hilbert-Schmidt operator (which is the norm we consider here), then

$$
E\left\|P\left(\sigma_{s}\right)\left(\left|v_{s}^{n}\right|^{\beta}-\left|v_{s}\right|^{\beta}\right)\right\|^{2} \leq E\left\|P\left(\sigma_{s}\right)\right\|_{\mathbb{L}}^{2}\left\|\left(\left|v_{s}^{n}\right|^{\beta}-\left|v_{s}\right|^{\beta}\right)\right\|^{2} \leq E\left\|P\left(\sigma_{s}\right)\right\|_{\mathbb{L}_{2}}^{2}\left\|\left(\left|v_{s}^{n}\right|^{\beta}-\left|v_{s}\right|^{\beta}\right)\right\|^{2},
$$

then by Holder inequality,

$$
\int_{t_{0}}^{t} E\left\|P\left(\sigma_{s}\right)\left(\left|v_{s}^{n}\right|^{\beta}-\left|v_{s}\right|^{\beta}\right)\right\|^{2} d s \leq M\left(t_{0}\right)^{2} \int_{t_{0}}^{t}\left\{E\left\|P\left(\sigma_{s}\right)\right\|_{\mathbb{L}_{2}}^{4}\right\}^{\frac{1}{2}} \cdot\left\{E\left\|\left|v_{s}^{n}\right|^{\beta}-\left|v_{s}\right|^{\beta}\right\|^{4}\right\}^{\frac{1}{2}} d s,
$$

by assumption of Model 2, $E\left\|P\left(\sigma_{s}\right)\right\|_{\mathbb{L}_{2}}^{4} \lesssim E\left\|\sigma_{s}\right\|^{4 d}$ is uniformly bounded for any $s \in I$, thus,

$$
E \|\left.\int_{t_{0}}^{t} G_{t-s} \Sigma_{s}\left(v_{s}^{n}-v_{s}\right) d W_{s}\right|^{2} \lesssim \int_{t_{0}}^{t}\left\{E\left\|\left|v_{s}^{n}\right|^{\beta}-\left|v_{s}\right|^{\beta}\right\|^{4}\right\}^{\frac{1}{2}} d s .
$$

Thus, we only need to consider $E \|\left|v_{s}^{n}\right|^{\beta}-\left.\left|v_{s}\right|^{\beta}\right|^{4}$.

$$
\begin{aligned}
E \|\left|v_{s}^{n}\right|^{\beta}-\left.\left|v_{s}\right|^{\beta}\right|^{4} & =\int_{\Omega}\left(\left.\int_{0}^{M}|| v_{s}^{n}\right|^{\beta}-\left.\left|v_{s}\right|^{\beta}\right|^{2} d x\right)^{2} d \mathbb{P} \leq \int_{\Omega}\left\{\left(\int_{0}^{M} 1^{\frac{1}{1-\beta}} d x\right)^{2(1-\beta)} \cdot\left[\left.\int_{0}^{M}|| v_{s}^{n}\right|^{\beta}-\left.\left|v_{s}\right|^{\beta}\right|^{\frac{2}{\beta}} d x\right]^{2 \beta}\right\} d \mathbb{P} \\
& \lesssim \int_{\Omega}\left[\left.\int_{0}^{M}|| v_{s}^{n}\right|^{\beta}-\left.\left|v_{s}\right|^{\beta}\right|^{\frac{2}{\beta}} d x\right]^{2 \beta} d \mathbb{P} \lesssim\left(\int_{\Omega} 1^{\frac{1}{1-2 \beta}} d \mathbb{P}\right)^{1-2 \beta} \cdot\left[\int_{\Omega}\left[\left.\int_{0}^{M}|| v_{s}^{n}\right|^{\beta}-\left.\left|v_{s}\right|^{\beta}\right|^{\frac{2}{\beta}} d x\right] d \mathbb{P}\right]^{2 \beta} \\
& \lessgtr\left[\int_{\Omega}\left[\left.\int_{0}^{M}|| v_{s}^{n}\right|^{\beta}-\left.\left|v_{s}\right|^{\beta}\right|^{\frac{2}{\beta}} d x\right] d \mathbb{P}\right]^{2 \beta} .
\end{aligned}
$$

Now, if we can bound $\int_{\Omega}\left[\left.\int_{0}^{M}|| v_{s}^{n}\right|^{\beta}-\left.\left|v_{s}\right|^{\beta}\right|^{\frac{2}{\beta}} d x\right] d \mathbb{P}$ by $E \| v_{s}^{n}-\left.v_{s}\right|^{2}$, then we are done. To do so, let's first consider the following lemma:

Lemma 3.2. If $a$, $b$ are two non-negative real numbers, and $0<\beta<1$, then $\left(\left|a^{\beta}-b^{\beta}\right|\right)^{\frac{2}{\beta}} \leq(a-b)^{2}$.

Proof. If any number is zero, or they are equal, then the inequality is trivially correct, thus, we assume that neither of them is zero. Moreover, we may assume that $a>b$. Set $p=\frac{1}{\beta}>1, x:=b^{\beta}$, $y:=(a-b)^{\beta}$. 
For $p>1$, it is clear that for any two positive numbers $x$ and $y,(x+y)^{p} \geq x^{p}+y^{p}$. Thus, $\left(b^{\beta}+(a-b)^{\beta}\right)^{p} \geq\left(b^{\beta}\right)^{p}+\left((a-b)^{\beta}\right)^{p}=a$, which implies that $b^{\beta}+(a-b)^{\beta} \geq a^{\beta}=>(a-b)^{\beta} \geq a^{\beta}-b^{\beta}$, which is exactly what we need.

Now, according to Lemma 3.2, $\left.|| v_{s}^{n}\right|^{\beta}-\left.\left|v_{s}\right|^{\beta}\right|^{\frac{2}{\beta}} \leq\left(\left|v_{s}^{n}\right|-\left|v_{s}\right|\right)^{2} \leq\left(v_{s}^{n}-v_{s}\right)^{2}$, thus,

$$
\int_{\Omega}\left[\left.\int_{0}^{M}|| v_{s}^{n}\right|^{\beta}-\left.\left|v_{s}\right|^{\beta}\right|^{\frac{2}{\beta}} d x\right] d \mathbb{P} \leq \int_{\Omega}\left[\int_{0}^{M}\left(v_{s}^{n}-v_{s}\right)^{2} d x\right] d \mathbb{P}=E\left\|v_{s}^{n}-v_{s}\right\|^{2} \leq\left\|v_{s}^{n}-v_{s}\right\|_{C}^{2} .
$$

Thus, we can conclude that the operator $\Psi$ is continuous, and if a fixed point of it exists, it must be a mild solution to Problem 3.1.

3.2.4. Step 4. In this part, let's first show that the stochastic volatility operator $\Sigma_{t}$ defined in either model satisfy a similar condition as the drift term $\Delta_{t}$ :

There exist two non-negative continuous functions $\tau_{1}(t)$ and $\tau_{2}(t)$ from $I$ to $[0, \infty)$ such that for any $t$ in $I$,

$$
E\left\|\Sigma_{t}\left(v_{t}\right)\right\|^{2} \leq \tau_{1}(t) E\left\|v_{t}\right\|^{2}+\tau_{2}(t) .
$$

We call such kind of condition: Condition $C$.

For Model 1:

$$
E\left\|\Sigma_{t}\left(v_{t}\right)\right\|^{2}=E\left\|\sigma_{t} \cdot\left|v_{t}\right|^{\beta}\right\|^{2} \leq\left(E\left\|\sigma_{t}\right\|^{4}\right)^{\frac{1}{2}}\left(E\left\|\left|v_{t}\right|^{\beta}\right\|^{4}\right)^{\frac{1}{2}} \lesssim\left(E\left\|\left|v_{t}\right|^{\beta}\right\|^{4}\right)^{\frac{1}{2}},
$$

since $E\left\|\sigma_{t}\right\|^{4}$ is uniformly bounded.

Consider

$$
\begin{array}{r}
\left(E\left\|\left|v_{t}\right|^{\beta}\right\|^{4}\right)^{\frac{1}{2}}=\left(\int_{\Omega}\left[\int_{0}^{M}\left|v_{t}(\omega, x)\right|^{2 \beta} d x\right]^{2} d \mathbb{P}\right) \leq\left(\int_{\Omega}\left[M^{2(1-\beta)} \cdot\left(\int_{0}^{M}\left|v_{t}\right|^{2}\right)^{2 \beta}\right] d \mathbb{P}\right) \\
\leq M^{(2-2 \beta)} \cdot\left(E\left\|v_{t}\right\|^{2}\right)^{\beta} \leq M^{(2-2 \beta)} \cdot\left(E\left\|v_{t}\right\|^{2}+1\right),
\end{array}
$$

the last inequality holds since if $E\left\|v_{1}\right\|^{2} \geq 1$, then $\left(E\left\|v_{t}\right\|^{2}\right)^{\frac{\beta}{2}} \leq E\left\|v_{1}\right\|^{2}$, otherwise, $\left(E\left\|v_{t}\right\|^{2}\right)^{\beta} \leq 1$.

For Model 2:

$$
E\left\|\Sigma_{t}\left(v_{t}\right)\right\|^{2}=E\left\|P\left(\sigma_{t}\right)\left(\left|v_{t}\right|^{\beta}\right)\right\|^{2} \leq\left(E\left\|P\left(\sigma_{t}\right)\right\|^{4}\right)^{\frac{1}{2}}\left(E\left\|\left|v_{t}\right|^{\beta}\right\|^{4}\right)^{\frac{1}{2}} \lesssim\left(E\left\|\left|v_{t}\right|^{\beta}\right\|^{4}\right)^{\frac{1}{2}},
$$

since $P(\cdot)$ is a polynomial of degree $d, E\left\|P\left(\sigma_{t}\right)\right\|^{4}$ can be bounded by a function of $E\left\|v_{t}\right\|^{4 d}$, which is uniformly bounded in Model 2. And for the rest part, we can simply copy the proof of Model 1.

Thus, we proved that both models satisfy Condition $C$.

The following Steps are inspired and modified from the proofs in [4], [19]. 
3.2.5. Step 5. In this part, we show the existence of a local mild solutions starting at point $t_{0} \in[0, T)$.

Choose $\delta_{1}>0$ such that $\left[t_{0}, t_{0}+\delta_{1}\right] \subset[0, T)$. Then set

$$
\begin{aligned}
& M\left(t_{0}\right)=\sup _{t \in\left[t_{0}, t_{0}+\delta_{1}\right]}\left\{\left\|G_{t}\right\|\right\}, \\
& R\left(t_{0}\right)=3 M\left(t_{0}\right)\left(3 E\left\|v_{t_{0}}\right\|^{2}+2\right), \\
& \Gamma_{1}\left(t_{0}\right)=\sup _{t \in\left[t_{0}, t_{0}+\delta_{1}\right]}\left\{E\left\|\Delta_{t}\left(v_{t}\right)\right\|^{2} \mid E\left\|v_{t}\right\|^{2} \leq R\left(t_{0}\right)\right\}, \\
& \Gamma_{2}\left(t_{0}\right)=\sup _{t \in\left[t_{0}, t_{0}+\delta_{1}\right]}\left\{E\left\|\Sigma_{t}\left(v_{t}\right)\right\|^{2} \mid E\left\|v_{t}\right\|^{2} \leq R\left(t_{0}\right)\right\},
\end{aligned}
$$

here we use the facts that $v_{t}$ is mean-squared continuous, and $\Delta_{t}, \Sigma_{t}$ satisfy Condition $C$.

Let $\Xi$ denotes $\left\{v_{t} \in C\left(I, L^{2}(\Omega, \mathbb{H})\right) \mid E\left\|v_{t}\right\|^{2} \leq R\left(t_{0}\right)\right\}$ which is a closed ball centered at 0 with radius $R\left(t_{0}\right)$.

By Lemma 2.1, we may assume that there exists a positive constant $\Theta$ such that for any $I$ :

$$
E\left\|\int_{t_{0}}^{t} G_{t-s} \Sigma_{s}\left(v_{s}\right) d W_{s}\right\|^{2} \leq \Theta M\left(t_{0}\right)^{2} \int_{t_{0}}^{t} E\left\|\Sigma_{s}\left(v_{s}\right)\right\|^{2} d s .
$$

Then set

$$
\delta=\min \left\{\delta_{1}, \frac{E\left\|v_{t_{0}}\right\|^{2}+1}{\Gamma_{1}\left(t_{0}\right)}, \frac{E\left\|v_{t_{0}}\right\|^{2}+1}{\Theta \Gamma_{2}\left(t_{0}\right)}\right\} .
$$

Then for any $v \in \Xi, t \in\left[t_{0}, t_{0}+\delta\right]$,

$$
\begin{array}{r}
E\left\|\Psi\left(v_{t}\right)\right\|^{2} \leq 3 E\left\|G_{t-t_{0}} v_{t_{0}}\right\|^{2}+3 E\left\|\int_{t_{0}}^{t} G_{t-s} \Delta_{s}\left(v_{s}\right) d s\right\|^{2}+3 E\left\|\int_{t_{0}}^{t} G_{t-s} \Sigma_{s}\left(v_{s}\right) d W_{s}\right\|^{2} \\
\leq 3 M\left(t_{0}\right)^{2} E\left\|v_{t_{0}}\right\|^{2}+3 M\left(t_{0}\right)^{2} \Gamma_{1}\left(t_{0}\right) \delta+3 M\left(t_{0}\right)^{2} \Theta \Gamma_{2}\left(t_{0}\right) \delta \\
\leq 3 M\left(t_{0}\right)^{2}\left(3 E\left\|v_{t_{0}}\right\|^{2}+2\right)=R\left(t_{0}\right),
\end{array}
$$

thus, $\Psi$ maps $\Xi$ into itself.

Then we show that $\Psi: \Xi \rightarrow \Xi$ is compact.

First, we show that $\left\{\Psi\left(v_{t}\right) \mid v_{t} \in \Xi\right\}$ is relatively compact in $L^{2}(\Omega, \mathbb{H})$ for any $t \in\left[t_{0}, t_{0}+\delta\right]$. This is clear for $t=t_{0}$ since there is only one point.

In general, for $t>t_{0}, 0<\varepsilon<t-t_{0}$, and $v_{t} \in \Xi$, define

$$
\begin{array}{r}
\Psi^{\varepsilon}\left(v_{t}\right)=G_{t-t_{0}} v_{t_{0}}+\int_{t_{0}}^{t-\varepsilon} G_{t-t_{0}} \Delta_{s}\left(v_{s}\right) d s+\int_{t_{0}}^{t-\varepsilon} G_{t-t_{0}} \Sigma_{s}\left(v_{s}\right) d W s \\
=G_{t-t_{0}} v_{t_{0}}+G_{\varepsilon} \int_{t_{0}}^{t-\varepsilon} G_{t-\varepsilon-t_{0}} \Delta_{s}\left(v_{s}\right) d s+G_{\varepsilon} \int_{t_{0}}^{t-\varepsilon} G_{t-\varepsilon-t_{0}} \Sigma_{s}\left(v_{s}\right) d W s,
\end{array}
$$

since $G_{t}$ is compact for any $t>0,\left\{\Psi\left(v_{t}\right) \mid v \in \Xi\right\}$ is also relatively compact in $L^{2}(\Omega, \mathbb{H})$, for any $\varepsilon$. 
Moreover, for any $v \in \Xi$, we have

$$
\begin{array}{r}
E\left\|\Psi\left(v_{t}-\Psi^{\varepsilon}\left(v_{t}\right)\right)\right\|^{2} \leq 3 E\left\|\int_{t-\varepsilon}^{t} G_{t-s} \Delta_{s}\left(v_{s}\right) d s\right\|^{2}+3 E\left\|\int_{t-\varepsilon}^{t} G_{t-s} \Sigma_{s}\left(v_{s}\right) d W_{s}\right\|^{2} \\
\leq 3 M\left(t_{0}\right)^{2} \cdot\left[\Gamma_{1}\left(t_{0}\right)+\Theta \Gamma_{2}\left(t_{0}\right)\right] \varepsilon,
\end{array}
$$

thus, $\left\{\Psi\left(v_{t}\right) \mid v \in \Xi\right\}$ is arbitrarily close to relatively compact sets in $L^{2}(\Omega, \mathbb{H})$. Thus, for any $t \in$ $\left[t_{0}, t_{0}+\delta\right],\left\{\Psi\left(v_{t}\right) \mid v \in \Xi\right\}$ is relatively compact.

Next, let's show that $\Psi(\Xi)$ is an equicontinuous family of functions in $C\left(I, L^{2}(\Omega, \mathbb{H})\right)$. have

For any $v \in \Xi, t_{0} \leq t_{1} \leq t_{2} \leq t_{0}+\delta$, apply Lemma 2.1 to $G_{t_{2}-s} \Sigma_{s}\left(v_{s}\right)$ and $\left[G_{t_{2}-s}-G_{t_{1}-s}\right] \Sigma_{s}\left(v_{s}\right)$, we

$$
\begin{array}{r}
E\left\|\Psi\left(v_{t_{1}}\right)-\Psi\left(v_{t_{2}}\right)\right\|^{2} \lesssim E\left\|G_{t_{2}-t_{0}} v_{t_{0}}-G_{t_{1}-t_{0}} v_{t_{0}}\right\|^{2}+E\left\|\int_{t_{1}}^{t_{2}} G_{t_{2}-s} \Delta_{s}\left(v_{s}\right) d s\right\|^{2} \\
+E\left\|\int_{t_{0}}^{t_{1}}\left[G_{t_{2}-s}-G_{t_{1}-s}\right] \Delta_{s}\left(v_{s}\right) d s\right\|^{2}+E\left\|\int_{t_{1}}^{t_{2}} G_{t_{2}-s} \Sigma_{s}\left(v_{s}\right) d W_{s}\right\|^{2} \\
+E\left\|\int_{t_{0}}^{t_{1}}\left[G_{t_{2}-s}-G_{t_{1}-s}\right] \Sigma_{s}\left(v_{s}\right) d W_{s}\right\|^{2} \\
\leq E\left\|\left[G_{t_{2}-t_{0}}-G_{t_{1}-t_{0}}\right] v_{t_{0}}\right\|^{2} d s+M\left(t_{0}\right)^{2}\left(t_{2}-t_{1}\right) \Gamma_{1}\left(t_{0}\right) \\
+M\left(t_{0}\right)^{2} \Gamma_{1}\left(t_{0}\right) \int_{t_{0}}^{t_{1}}\left\|G_{t_{2}-s}-G_{t_{1}-s}\right\|^{2} d s+\Theta M\left(t_{0}\right)^{2}\left(t_{2}-t_{1}\right) \Gamma_{2}\left(t_{0}\right) \\
+\Theta M\left(t_{0}\right)^{2} \Gamma_{2}\left(t_{0}\right) \int_{t_{0}}^{t_{1}}\left\|G_{t_{2}-s}-G_{t_{1}-s}\right\|^{2} d s
\end{array}
$$

let's denote the five components of the right hand side of the last inequality above sequentially as (1), (2), (3), (4) and (5).

Since $G_{t-t_{0}} v_{t_{0}}$ is continuous on $\left[t_{0}, t_{0}+\delta\right]$, it is uniformly continuous, thus, (1) $\rightarrow 0$, as $\left(t_{2}-t_{1}\right) \rightarrow 0$; Obviously, (2) and (4) tend to 0 if $\left(t_{2}-t_{1}\right) \rightarrow 0$. Now notice that $G_{t}$ is compact for $t>0$, we know that $G_{t}$ is continuous in operator norm. Combining this fact with Lebesgue Dominated Convergence Theorem, we know that (3) and (5) also go to 0 if $\left(t_{2}-t_{1}\right) \rightarrow 0$. Thus, $\Psi: \Xi \rightarrow \Xi$ is equicontinuous.

Now, we are ready to show the existence of local mild solution.

By Leray-Schauder Fixed Point Theorem, there exists a fixed point, thus, a mild solution to Problem $(3.1)$ over $\left[t_{0}, t_{0}+\delta\right]$ with given initial value $v_{t_{0}}=v_{0}$. In particular, $t_{0}$ can be set to 0 .

Replace $t_{0}$ by $t_{0}+\delta, v_{t_{0}}$ by $v_{t_{0}+\delta}$, there exists $\widetilde{\delta}>0$ such that we can extend the solution to $\left[t_{0}, t_{0}+\delta+\widetilde{\delta}\right]$. Repeat this process, in the end, we can get a maximal semi-closed interval $\left[0, T_{1}\right) \subset$ $[0, T]$ over which a mild solution exists. 
In the following two steps, we will show that $\left[0, T_{1}\right)$ can be extended to $[0, T]$, and, thus, get the mild solution we want.

3.2.6. Step 6. In this part, we show that if $\left[0, T_{1}\right]$ cannot be extended, then $\lim _{t \rightarrow T_{1}^{-}} E\left\|v_{t}\right\|^{2}=\infty$.

First, we show that if $\left[0, T_{1}\right]$ cannot be extended, then $\lim \sup E\left\|v_{t}\right\|^{2}=\infty$. If not, then there exits a constant $0<r\left(T_{1}\right)<\infty$ such that $\sup _{t \rightarrow T_{1}^{-}} E\left\|v_{t}\right\|^{2}<r\left(T_{1}\right)$.

$$
t \rightarrow T_{1}^{-}
$$

Denote

$$
\begin{aligned}
& M\left(T_{1}\right)=\sup _{0<t<T_{1}+\delta_{2}}\left\|G_{t}\right\| \\
& \Gamma_{1}\left(T_{1}\right)=\sup _{0<t<T_{1}+\delta_{2}}\left\{E\left\|\Delta_{t}\left(v_{t}\right)\right\|^{2} \mid E\left\|v_{t}\right\|^{2} \leq r\left(T_{1}\right)\right\} \\
& \Gamma_{2}\left(T_{2}\right)=\sup _{0<t<T_{1}+\delta_{2}}\left\{E\left\|\Sigma_{t}\left(v_{t}\right)\right\|^{2} \mid E\left\|v_{t}\right\|^{2} \leq r\left(T_{1}\right)\right\}
\end{aligned}
$$

where $\delta_{2}$ is chosen so that $T_{1}+\delta_{2} \leq T$. For $0<t^{1}<t^{2}<T_{1}$,

$$
\lim _{t^{1}, t^{2} \rightarrow T_{1}^{-}} E\left\|v_{t^{1}}-v_{t^{2}}\right\|^{2}=0
$$

since

$$
\begin{array}{r}
E\left\|v_{t^{1}}-v_{t^{2}}\right\|^{2} \lesssim\left\|G_{t^{1}}-G_{t^{2}}\right\|^{2} E\left\|v_{t_{0}}\right\|^{2}+M\left(T_{1}\right)^{2} \Gamma_{1}\left(T_{1}\right)\left(t^{2}-t^{1}\right)+M\left(T_{1}\right)^{2} \Gamma_{1}\left(T_{1}\right) \int_{0}^{t^{1}}\left\|v_{t^{1}}-v_{t^{2}}\right\|^{2} d s \\
+\Theta M\left(T_{1}\right)^{2} \Gamma_{2}\left(T_{1}\right)\left(t^{2}-t^{1}\right)+\Theta M\left(T_{1}\right)^{2} \Gamma_{2}\left(T_{1}\right) \int_{0}^{t^{1}}\left\|v_{t^{1}}-v_{t^{2}}\right\|^{2} d s,
\end{array}
$$

the right hand side above tends to zero due to the continuity of $G_{t}$ in the uniform operator topology for $t>0$ which follows from the compactness of $G_{t}$. Thus, $\lim _{t \rightarrow T_{1}^{-}} E\left\|v_{t}\right\|^{2}$ exists.

Now, let's show that if $\left[0, T_{1}\right)$ cannot be extended, $\lim _{t \rightarrow T_{1}^{-}} E\left\|v_{t}\right\|^{2}=\infty$.

If not, then there exits a constant $0<r<\infty$ and a sequence $t_{n} \rightarrow T_{1}$ such that $E\left\|v_{t_{n}}\right\|^{2}<r$.

\section{Denote}

$$
\begin{aligned}
& \widetilde{M}=\sup _{0<t<T_{1}}\left\|G_{t}\right\| \\
& \widetilde{\Gamma}_{1}=\sup _{0<t<T}\left\{E\left\|\Delta_{t}\left(v_{t}\right)\right\|^{2} \mid E\left\|v_{t}\right\|^{2} \leq 3 \widetilde{M}^{2}(r+1)\right\} \\
& \widetilde{\Gamma}_{2}=\sup _{0<t<T}\left\{E\left\|\Sigma_{t}\left(v_{t}\right)\right\|^{2} \mid E\left\|v_{t}\right\|^{2} \leq 3 \widetilde{M}^{2}(r+1)\right\}
\end{aligned}
$$


Now, $t \mapsto E\left\|v_{t}\right\|^{2}$ is continuous and $\lim _{t \rightarrow T_{1}^{-}} E\left\|v_{t}\right\|^{2}=\infty$, thus we can find $\left\{l_{n}\right\}_{n}$ such that $0<l_{n}<T-t_{n}$, $E\left\|v_{t}\right\|^{2} \leq 3 \widetilde{M}^{2}(r+1)$ for any $t \in\left[t_{n}, t_{n}+l_{n}\right]$ and $E\left\|v_{t_{n}+l_{n}}\right\|^{2}=3 \widetilde{M}^{2}(r+1)$.

Then

$$
\begin{aligned}
3 \widetilde{M}^{2}(r+1)=E\left\|v_{t_{n}+l_{n}}\right\|^{2} \leq 3 E\left\|G_{t_{n}} v_{t_{n}}\right\|^{2} & +3 E\left\|\int_{t_{n}}^{t_{n}+l_{n}} G_{t_{n}+l_{n}-s} \Delta_{s}\left(v_{s}\right) d s\right\|^{2} \\
& +3 E\left\|\int_{t_{n}}^{t_{n}+l_{n}} G_{t_{n}+l_{n}-s} \Sigma_{s}\left(v_{s}\right) d W_{s}\right\|^{2} \\
& <3 \widetilde{M}^{2} r+3 \widetilde{M}^{2} \widetilde{\Gamma}_{1} l_{n}+3 \Theta \widetilde{M}^{2} \widetilde{\Gamma}_{2} l_{n},
\end{aligned}
$$

and the right hand side of the last inequality tends to 0 as $n \rightarrow \infty$, which implies that $\widetilde{M}$ is less than 0 , contradiction.

Thus, we can conclude that if $E\left\|v_{T_{1}}\right\|^{2}<\infty$, then $\left[0, T_{1}\right)$ can be extended.

3.2.7. Step 7. In this part, we show that under the assumptions of our models $E\left\|v_{T_{1}}\right\|^{2}<\infty$, thus, conclude that the mild solution is defined across the whole $[0, T]$.

Assume that $T_{1} \neq T$,

$$
\begin{aligned}
& \bar{M}=\sup _{t \in[0, T]}\left\|G_{t}\right\|^{2} \\
& s_{1}=\max _{t \in[0, T]} f_{1} \\
& s_{2}=\max _{t \in[0, T]} f_{2} \\
& s_{3}=\max _{t \in[0, T]} \tau_{1} \\
& s_{4}=\max _{t \in[0, T]} \tau_{2} .
\end{aligned}
$$

Then

$$
\begin{array}{r}
E\left\|v_{T_{1}}\right\|^{2} \leq 3 E\left\|G_{T_{1}} v_{0}\right\|^{2}+3 E \mid \int_{0}^{T_{1}} G_{T_{1}-s} \Delta_{s}\left(v_{s}\right) d s\left\|^{2}+3 E\right\| \int_{0}^{T_{1}} G_{T_{1}-s} \Sigma_{s}\left(v_{s} d W_{s}\right) \|^{2} \\
\leq 3 \bar{M}^{2} E\left\|v_{0}\right\|^{2}+3 \bar{M} \int_{0}^{T_{1}} E\left\|\Delta_{s}\left(v_{s}\right)\right\|^{2} d s+3 \Theta \bar{M} \int_{0}^{T_{1}} E\left\|\Sigma_{s}\left(v_{s}\right)\right\|^{2} d s \\
\leq\left\{3 \bar{M}^{2} E\left\|v_{0}\right\|^{2}+3 \bar{M}^{2} s_{2} T_{1}+3 \Theta \bar{M}^{2} s_{4} T_{1}\right\}+\left\{3 \bar{M}^{2} s_{1}+3 \Theta \bar{M}^{2} s_{3}\right\} \cdot \int_{0}^{T_{1}} E\left\|v_{s}\right\|^{2} d s,
\end{array}
$$

denote $\left\{3 \bar{M}^{2} E\left\|v_{0}\right\|^{2}+3 \bar{M}^{2} s_{2} T_{1}+3 \Theta \bar{M}^{2} s_{4} T_{1}\right\}$ as $\Pi_{1},\left\{3 \bar{M}^{2} s_{1}+3 \Theta \bar{M}^{2} s_{3}\right\}$ as $P i_{2}$, then by Gronwall's inequality, we get

$$
E\left\|v_{T_{1}}\right\|^{2} \leq \Pi_{1} \cdot e^{\Pi_{2} T_{1}}<\infty .
$$

Thus, by the result of Step 6, we have shown the existence of mild solution to Problem (3.1). 


\section{A Special Case: Ornstein-Uhlenbeck Process}

In our models, there is a quite unnatural condition: $E\left\|\Sigma_{t}\right\|^{p}$ is uniformly bounded where $p \geq 4$. Usually an assumption on the existence of second moment is more realistic, however, due to the complicated correlation between the interest rate process and the stochastic volatility process, to achieve such a goal, we need to put stronger constrains. Two possible ways are:

1) Every sample path of $\Sigma_{t}$ is bounded by some fixed constant.

2) $\mathbb{P}\left(\left\|\Sigma_{t}\right\|^{2} \geq N\right)$ decays fast enough as $N \rightarrow \infty$.

Notice that if 1 ) is true, then clearly $E\left\|\Sigma_{t}\right\|^{2}$ is uniformly bounded. However, this is still a too strong condition. In the following, let's focus on case 2).

In general, 2) is not satisfied, however in the case of an Ornstein-Uhlenbeck process, under some natural condition, it can be achieved. Let's first consider Model 1 to illustrate the idea.

Assume that the process $\sigma_{t}$ satisfies:

$$
d \sigma_{t}=\bar{A} \sigma_{t}+d \bar{W}_{t}
$$

where $\bar{W}_{t}$ is a $\bar{Q}$-Wiener process valued in real separable Hilbert space $\bar{U}$ and $\bar{A}$ generates strongly continuous contraction semigroup: $\bar{G}_{t}$. Then $\sigma_{t}=\bar{G} t \sigma_{0}+\int_{0}^{t} \bar{G}_{t-s} d \bar{W}_{s}$, and $\int_{0}^{t} \bar{G}_{t-s} d \bar{W}_{s}$ is continuous martingale. Its local covariance operator $\bar{Q}_{t}$ is $\bar{G}_{t} \bar{Q} \bar{G}_{t}^{*}$, and notice that $\operatorname{Tr} \bar{Q}_{t}=\left\|\bar{G}_{t}\right\|_{\mathbb{I}_{2}\left(\bar{U}_{\bar{Q}}, \mathbb{L}_{2}(\mathbb{L}, \mathbb{R})\right)}^{2} \leq$ $\left\|\bar{G}_{t}\right\|^{2} \operatorname{Tr}(\bar{Q}) \leq \operatorname{Tr}(\bar{Q})$, which is obviously finite.

The following lemma will be used to control the decay of tail probability of process $\Sigma_{t}$.

Lemma 4.1. (Lemma 6.2 Chapter 7 [5]) Let $M_{t}$ be a continuous $H$-valued martingale over [0,T] with the local covariance operator $Q_{t}$ such that

$$
\sup _{0 \leq t \leq T} \operatorname{Tr} Q_{t} \leq N, \text { a.s., }
$$

for some $N>0$. Then the following estimate holds

$$
\mathbb{P}\left\{\sup _{0 \leq s \leq t}\left\|M_{s}\right\| \geq r\right\} \leq \exp \left\{-\frac{r^{2}}{4 N t}\right\},
$$

for any $t \in(0, T]$ and $r>0$.

Notice that our construction of $\sigma_{t}$ in this section satisfies conditions of Lemma 4.1.

Based on the proof of the main theorem, for Model 1, we only need to estimate the expectation in the following form

$$
E\left\|\sigma_{t}\left|v_{t}\right|^{\beta}\right\|_{\mathbb{L}_{2}}^{2}=E\left[\left\|\sigma_{t}\right\|_{\mathbb{L}_{2}}^{2} \cdot\left\|\left|v_{t}\right|^{\beta}\right\|_{\mathbb{H}}^{2}\right] \leq 2 E\left[\left\|\bar{G}_{t} \sigma_{0}\right\|_{\mathbb{L}_{2}}^{2} \cdot\left\|\left|v_{t}\right|^{\beta}\right\|_{\mathbb{H}}^{2}\right]+2 E\left[\left\|\int_{0}^{t} \bar{G}_{t-s} d \bar{W}_{s}\right\|_{\mathbb{L}_{2}}^{2} \cdot\left\|\left|v_{t}\right|^{\beta}\right\|_{\mathbb{H}}^{2}\right],
$$


clearly $2 E\left[\left\|\bar{G}_{t} \sigma_{0}\right\|_{\mathbb{L}_{2}}^{2} \cdot\left\|\left|v_{t}\right|^{\beta}\right\|_{\mathbb{H}}^{2}\right]$ is finite. For the other component, we can apply Lemma 4.1. By our construction, there exists positive constant $N>0$ which is a upper bound of the local covariance operator, thus

$$
\mathbb{P}\left\{\sup _{0 \leq s \leq T}\left\|M_{s}\right\| \geq r\right\} \leq \exp \left\{-\frac{r^{2}}{4 N T}\right\}
$$

where $M_{t}=\int_{0}^{t} \bar{G}_{t-s} d \bar{W}_{s}$.

Set $E_{i}=\left\{\omega \in \Omega \mid i \leq \sup _{t \in[0, T]}\left\|M_{t}\right\|<i+1\right\}$ to be an event set, $q_{i}=\mathbb{P}\left(E_{i}\right)$, for $i=1,2,3, \ldots, n, \ldots$, then $\sum_{i=1}^{\infty} q_{i}=1$. Thus, $E\left[\left\|\int_{0}^{t} \bar{G}_{t-s} d \bar{W}_{s}\right\|_{\mathbb{L}_{2}}^{2} \cdot\left\|\left|v_{t}\right|^{\beta}\right\|_{\mathbb{H}}^{2}\right]=\sum_{i=1}^{\infty} E\left\{\left\{\left\|M_{t}\right\|_{\mathbb{L}_{2}}^{2} \cdot\left\|\left|v_{t}\right|^{\beta}\right\|_{\mathbb{H}}^{2}\right\} \mid E_{i}\right\} \cdot q_{i}$.

Then

$$
\begin{array}{r}
E\left[\left\|\int_{0}^{t} \bar{G}_{t-s} d \bar{W}_{s}\right\|_{\mathbb{L}_{2}}^{2} \cdot\left\|\left|v_{t}\right|^{\beta}\right\|_{\mathbb{H}}^{2}\right] \leq \sum_{i=1}^{\infty}(i+1)^{2} E\left[\left\|\left|v_{t}\right|^{\beta} \mid E_{i}\right\|_{\mathbb{H}}^{2}\right] \cdot q_{i} \\
=\sum_{i=1}^{\infty}(i+1)^{2} \sqrt{q_{i}} \cdot E\left[\left\|\left|v_{t}\right|^{\beta} \mid E_{i}\right\|_{\mathbb{H}}^{2}\right] \cdot \sqrt{q_{i}} \\
\leq \sum_{i=1}^{\infty}(i+1)^{2} \sqrt{3 \exp \left\{-\frac{i^{2}}{4 N T}\right\}} \cdot E\left[\left\|\left|v_{t}\right|^{\beta} \mid E_{i}\right\|_{\mathbb{H}}^{2}\right] \cdot \sqrt{q_{i}} \\
\leq \sum_{i=1}^{\infty}(i+1)^{2} \sqrt{3 \exp \left\{-\frac{i^{2}}{4 N T}\right\}} \cdot \sqrt{E\left[\left\|\left|v_{t}\right|^{\beta} \mid E_{i}\right\|_{\mathbb{H}}^{4}\right]} \cdot \sqrt{q_{i}} .
\end{array}
$$

Notice that $\bar{C}:=\sum_{i=1}^{\infty}\left[(i+1)^{2} \sqrt{3 \exp \left\{-\frac{i^{2}}{4 N T}\right\}}\right]^{2}<\infty$, and

$$
\sum_{i=1}^{\infty}\left[\sqrt{E\left[\left\|\left|v_{t}\right|^{\beta} \mid E_{i}\right\|_{\mathbb{H}}^{4}\right.} \cdot \sqrt{q_{i}}\right]^{2}=E\left[\left\|\left|v_{t}\right|^{\beta}\right\|_{\mathbb{H}}^{4}\right] \lesssim E\left[\left\|\left|v_{t}\right|^{2}\right\|_{\mathbb{H}}\right]^{\beta} \leq \infty,
$$

see Step 4 in the proof of the main theorem.

Thus, we can apply Cauchy-Schwartz inequality for countably infinite sum, and get

$$
\begin{gathered}
\left.E\left[\left\|\int_{0}^{t} \bar{G}_{t-s} d \bar{W}_{s}\right\|_{\mathbb{L}_{2}}^{2} \cdot\left\|\left|v_{t}\right|^{\beta}\right\|_{\mathbb{H}}^{2}\right] \leq \sqrt{\bar{C}} \cdot \sqrt{E\left[\left\|\left|v_{t}\right|^{\beta}\right\|_{\mathbb{H}}^{4}\right.}\right] \\
\quad \lesssim E\left[\left\|\left|v_{t}\right|^{\beta}\right\|_{\mathbb{H}}^{4}\right]^{\frac{1}{2}} \lesssim E\left[\left\|\left|v_{t}\right|^{2}\right\|_{\mathbb{H}}\right]^{\frac{\beta}{2}} \lesssim E\left[\left\|\left|v_{t}\right|^{2}\right\|_{\mathbb{H}}\right]+1,
\end{gathered}
$$

thus, we can get all the necessary estimations. Moreover, replace $\left|v_{t}\right|^{\beta}$ by $\left.|| v_{t}\right|^{\beta}-\left|v_{t_{n}}{ }^{\beta}\right|$, we can show that the map $\Psi$ is still continuous.

For Model 2, we essentially use the same ideas and proofs as above, with the exception that $E\left\|\sigma_{t}\right\|_{\mathbb{L}_{2}}^{2}$ must be replaced by $E\left\|P\left(\sigma_{t}\right)\right\|_{\mathbb{L}_{2}}^{2}$ which is then bounded from above by a function of $E\left\|\sigma_{t}\right\|_{\mathbb{L}_{2}}^{2 d}$ where $d=$ dgree of $P$. This allows us to reduce $p$ to $2 d$ in this special case. 


\section{Regularities}

In the general case, it is not easy to discuss regularity of the mild solutions. In this section, inspired by [3] and [6], we consider a very special model with simple stochastic volatility operators and show some related regularity properties.

Let's first state the model:

$$
\mathbb{H}_{k}=L^{2}\left([0,1], e^{\frac{2 x}{k}} d x\right), k>0
$$

$A_{k}=k \frac{\partial^{2}}{\partial x^{2}}+\frac{\partial}{\partial x}$ on $\mathbb{H}_{k}$, and the nth eigenfunction of $A_{k}$ is $e_{n}=\sqrt{2} \sin (n \pi x) e^{-\frac{x}{k}}$ while the nth eigenvalue is $-\lambda_{n}$ with $\lambda_{n}=\frac{1}{2 k}\left(1+\pi^{2} k^{2} n^{2}\right)$;

$W_{k}(t): \mathbb{H}_{k}$-valued, $Q_{k}$-Wiener process such that

$$
W_{k}(t)=\sum_{i=1}^{\infty} \sqrt{\phi_{i}} W_{k}^{i}(t) e_{i}, \operatorname{Tr} Q_{k}=\sum_{i=1}^{\infty} \phi_{i}<\infty,
$$

where $\left\{W_{k}^{n}(t)\right\}_{n=1}^{\infty}$ are independent identical one-dimensional Brownian motion.

The Model is

$$
g_{t}(x)=\sum_{n=1}^{\infty} \sqrt{\phi_{n}} g_{n}(t) e_{n}(x)
$$

where

$$
d g_{n}(t)=-\lambda_{n} g_{n} d t+\left|\sigma_{n}(t)\right| d W_{k}^{n}(t)
$$

with $g_{n}(0)$ and $g(0)$, all being deterministic and smooth enough, for instance, where $\sigma_{n}=\frac{1}{n^{\zeta}} f_{n}(t)$, and

$$
d f_{n}(t)=b_{n}\left(t, f_{n}(t)\right) d t+\eta_{n}\left(t, f_{n}(t)\right) d B_{n}(t)
$$

such that

1). $b_{n}$ and $\eta_{n}$ satisfy the standard linear growth and Lipschitz conditions and put some uniform bound on the initial values: for any $n, E\left\|f_{n}(0)\right\|^{2} \leq \bar{O}$.

2). $\left\{B_{n}(t)\right\}_{n=1}^{\infty}$ are also independent identical one-dimensional Brownian motion, and, together with $\left\{W_{k}^{n}(t)\right\}_{n=1}^{\infty}$, they generates the filtration.

Then we can immediately conclude that 
1). There exists a constant $\widetilde{O}>0$ such that for any $t, n, E\left\|f_{n}(t)\right\|^{2} \leq \widetilde{O}$;

2). $E\left[\int_{0}^{t} e^{-\lambda_{n}(t-s)}\left|f_{n}\right| d W_{n}^{k}\right]^{2} \leq \widetilde{O} \int_{0}^{t} e^{-2 \lambda_{n}(t-s)} d s=\widetilde{O}\left(\frac{1-e^{-\lambda_{n} t}}{2 \lambda_{n}}\right)$ by Ito isometry and 1$)$.

Now, let's investigate our model.

5.1. In this first part, let's show that $g_{t}$ is well-defined.

It's clear that $g_{t}$ is $\mathcal{F}_{t}$-adapted and continuous (notice that this is due to the fact that we can always replace the resulting process by their continuous version if necessary), thus, we only need to show that for any $t, g_{t} \in L^{2}(\Omega, \mathbb{H})$. For simplicity, without loss of generality (WLOG), we may assume that $g_{t}(0)=0$. Then for any $t$, assume that either $Q_{k}=I$ or $\operatorname{Tr}\left(Q_{k}\right)<\infty$, then

$$
\begin{array}{r}
E\left\|g_{t}\right\|_{\mathbb{H}_{k}}^{2}=E\left\|\sum_{n=1}^{\infty} \sqrt{\phi_{n}} g_{n}(t) e_{n}(x)\right\|_{\mathbb{H}_{k}}^{2}=\sum_{n=1}^{\infty} \phi_{n} E\left[g_{n}(t)^{2}\right]=\sum_{n=1}^{\infty} \phi_{n} E\left[\int_{0}^{t} e^{-2 \lambda_{n}(t-s)}\left|\sigma_{n}(s)\right| d W_{n}^{k}(s)\right]^{2} \\
\leq \sum_{n=1}^{\infty} \phi_{n} \frac{\widetilde{O}}{n^{2 \zeta}} \frac{1}{2 \lambda_{n}},
\end{array}
$$

which is finite under our assumptions.

5.2. Assume that either $Q_{k}=I$ or $\operatorname{Tr} Q_{k}<\infty$, then for any $t, g_{t}(x)$ as a function of $x$, with probability one, is $k$ times differentiable, $k<\zeta$.

Proof. For simplicity, WLOG, assume that $g_{0}=0$. Soblev Space $H^{\zeta}\left([0,1], e^{\frac{2 x}{k}} d x\right)$ is defined as

$$
h \in H^{\zeta} \Longleftrightarrow h \in \mathbb{H}_{k} \text { and }\left|h \|_{H^{\zeta}}^{2}=\sum_{n=1}^{\infty} n^{2 \zeta} \cdot\right|<h, e_{n}>\left.\right|^{2}<\infty \text {. }
$$

Now

$$
\begin{array}{r}
E\left\|g_{t}\right\|_{H^{\zeta}}^{2}=E\left\{\sum_{n=1}^{\infty} \phi_{n} n^{2 \zeta}\left|g_{n}(t)\right|^{2}\right\}=\sum_{n=1}^{\infty} n^{2 \zeta} E\left[\left|g_{n}(t)\right|^{2}\right] \phi_{n} \\
=\sum_{n=1}^{\infty} \phi_{n} E\left[\int_{0}^{t} e^{-\lambda_{n}(t-s)}\left|f_{n}(s)\right| d W_{n}^{k}(s)\right]^{2} \leq \sum_{n=1}^{\infty} \phi_{n} \bar{O} \frac{1}{2 \lambda_{n}}<\infty .
\end{array}
$$

5.3. (This is a modified version of Lemma 5.21 Chapter 5 [6]) If $\operatorname{Tr} Q_{k}<\infty$ or $\sum_{n=1}^{\infty} \frac{\phi_{n}}{n^{2 \zeta} \lambda_{n}^{(1-\gamma)}}<\infty$, $\gamma \in(0,1)$, then for any $x$ and $y \in[0,1]$,

$$
E\left|g_{t}(x)-g_{t}(y)\right|^{2} \lesssim|x-y|^{2 \gamma},
$$

in particular, if $\gamma>\frac{1}{2}$, then $g_{t}$ is $\frac{2 \gamma-1}{2}$-Hölder continuous by Kolmogorov's test for random fields. 
Proof. For any $n, e_{n}=\sqrt{2} \sin (n \pi x) e^{-\frac{x}{k}}$, thus, it's clear that there exits $\kappa>0$ such that

$$
\left|e_{n}(x)\right| \leq \kappa,\left|\nabla e_{n}(x)\right| \leq \kappa \lambda_{n}^{\frac{1}{2}},
$$

thus, $\left|e_{n}(x)-e_{n}(y)\right| \leq \kappa \lambda_{n}^{\frac{1}{2}}|x-y|$.

Then one can show that

$$
\left|e_{n}(x)-e_{n}(y)\right| \leq \kappa 2^{1-\gamma} \lambda_{n}^{\frac{\gamma}{2}}|x-y|^{\gamma}
$$

Now notice that

$$
\begin{array}{r}
g(t)=\sum_{n=1}^{\infty} \sqrt{\phi_{n}} g_{n}(t) e_{n}(t)=\sum_{n=1}^{\infty} \sqrt{\phi_{n}}\left[g_{n}(0) e^{-\lambda_{n} t} e_{n}(x)+\int_{0}^{t}\left|\sigma_{n}(s)\right| e^{-\lambda_{n}(t-s)} d W_{k}^{n}(s)\right] \\
=\sum_{n=1}^{\infty} \sqrt{\phi_{n}} g_{n}(0) e^{-\lambda_{n} t} e_{n}(x)+\sum_{n=1}^{\infty} \sqrt{\phi_{n}} \int_{0}^{t}\left|\sigma_{n}(s)\right| e^{-\lambda_{n}(t-s)} d W_{k}^{n}(s)=: I+I I,
\end{array}
$$

where $I$ represents the drift part and $I I$ represents the diffusion part. Clearly the drift part is smooth. Then, to investigate regularity, we only need to consider the diffusion part of our model

$$
E\left|g_{t}(x)-g_{t}(y)\right|^{2}=E\left[\left(\sum_{n=1}^{\infty} \sqrt{\phi_{n}} g_{n}(t)\left(e_{t}(x)-e_{t}(y)\right)\right)^{2}\right]
$$

However, for any $n, E\left[g_{n}(t)\right]=0$ (notice that this is only used when $g_{n}(0)$ are not all zero), indeed, since we assume that $g_{n}=0$ which implies that $g_{n}$ is a martingale, and also notice that for any $n \neq m$, $g_{n}(t) \perp g_{m}(t)$ since all the one-dimensional Brownian motions are independent with each other. Thus,

$$
\begin{array}{r}
E\left[\left(\sum_{n=1}^{\infty} \sqrt{\phi_{n}} g_{n}(t)\left(e_{t}(x)-e_{t}(y)\right)\right)^{2}\right]=\sum_{n=1}^{\infty} \phi_{n} \cdot\left|e_{n}(x)-e_{n}(y)\right|^{2} \cdot E\left(g_{n}^{2}(t)\right) \\
\leq \sum_{n=1}^{\infty} \phi_{n} \cdot \kappa^{2} \cdot 4^{1-\gamma} \lambda_{n}^{\gamma}|x-y|^{2 \gamma} \cdot \frac{1}{2 n^{2 \zeta} \lambda_{n}}=2^{1-2 \gamma} \cdot \kappa^{2}\left(\sum_{n=1}^{\infty} \frac{\phi_{n}}{n^{2 \zeta} \lambda_{n}^{1-\gamma}}\right) \cdot|x-y|^{2 \gamma} \\
\lesssim|x-y|^{2 \gamma} .
\end{array}
$$

Based on sections 5.2 and 5.3, we can see that if $\operatorname{Tr} Q_{k}<$ inf or $Q_{k}=I$, the mild solution is indeed a strong one, which inspires us to develop efficient numerical methods for implementation in the coming papers. 


\section{Conclusion}

We showed the existence of mild solution to the Cauchy problem of Hilbert-space valued stochastic differential equations with stochastic volatility operators, modeling the evolution of instantaneous forward rate curves. Moreover, the stochastic volatility operator processes we proposed here related the volatility operators with the level of the forward rates to the power of some fraction number $\beta<0.5$ ( $\beta<1$ under certain conditions ), which are analogs to the well-known SABR model in finite dimension case. By examining some specific models, we showed regularity properties of the solutions with the help of a second order differential operator as the regularity term. As mentioned in [18], the regularizing term $D(x) \frac{\partial^{2}}{\partial x^{2}}$ has strong physical meaning. In particular, $D(x)$ represents a "force" to dampen the curvature of the interest rate curve in the long run and reduce its oscillation. It is technically not "risk-neutral" but its presence, which is confirmed statistically, cannot really be arbitraged, due to the bid/ask spread.

Let's discuss here some potential generalizations and applications of our two models.

We believe that the kind of methodology introduced in this article - Hilbert spaced-valued stochastic processes with stochastic volatility operator - has applications in much wider areas beyond financial math. For instance, it could be applied to the equations modeling the temperature of complex systems. Leray-Schauder theorem, following the proof of a priori inequality, is a very powerful tool that has been applied in other areas and has numerous fields of application to be explored in the context of infinite dimensional stochastic processes.

Numerical implementation is crucial for applied science. Appropriate numerical methods need to be designed to implement the model. One of the state-of-art technique that people usually apply to solve this type of problem is the Galerkin method [9] which uses finite sub-space to approximate the infinite Hilbert space. However, most of the present research only deals with deterministic volatility operator case. Thus, a study on this direction would raise people's interest from both practical and theoretical aspects.

Third, notice that in our present model the second order differential operator $D(x) \frac{\partial^{2}}{\partial x^{2}}$ is timeindependent. However, due to its physical interpretation, there is no reason to keep this constrain, and in fact there is strong empirical evidence to support the time-dependence assumption. In a forthcoming paper, we envision to study the case of a time-dependent second order differential operator $D(t, x) \frac{\partial^{2}}{\partial x^{2}}$. Our results can be easily generalized to this situation under certain mild conditions.

\section{REFERENCES}

[1] Benth, F., Cathrine Simonsen, I. (2017): The Heston Stochastic Volatility Model in Hilbert Space. Stochastic Analysis and Applications 36, 733-750

[2] Carmona, R., Tehranchi, M, R. (2007): Interest Rate Models: An Infinite Dimensional Stochastic Analysis Perspective: An Infinite-dimensional Stochastic Analysis Perspective (Springer Finance). Springer

[3] Cont, R. (2005): Modeling Term Structure Dynamics: an infinite dimensional approach. International Journal of Theoretical and Applied Finance 8, 357-380

[4] Chen, P., Li, Y., Zhang, X. (2015): On the Initial Value Problem of Fractional Stochastic Evolution Equations in Hilbert Spaces. Communications on Pure \& Applied Analysis 15, 1817-1840

[5] Chow, P-L. (2014): Stochastic Partial Differential Equations (Advances in Applied Mathematics) 2nd edition. Chapman and Hall/CRC 
[6] Da Prato, G., Zabczyk, J. (2014): Stochastic Equations in Infinite Dimensions (Encyclopedia of Mathematics and its Applications) 2nd Edition. Cambridge University Press

[7] Douady, R. (1999): Model Calibration in Monte-Carlo Framework. Monte Carlo Methodologies and Applications for Pricing and Risk Management, Risk Books

[8] Douady, R. (2013): The Volatility of Low Rates. Riskdata working paper

[9] Douady, R. (2014): Yield Curve Smoothing and Residual Variance of Fixed Income Positions. Inspired by Finance. Springer, Cham

[10] Engle, K-J., Nagel, R. (1999): One-Parameter Semigroups for Linear Evolution Equations (Graduate Texts in Mathematics). Springer

[11] Filipović, D. (2001): Consistency Problems for Heath-Jarrow-Morton Interest Rate Models (Lecture Notes in Mathematics 1760). Springer

[12] Filipović, D., Teichmann, J. (2003): Existence of Invariant Manifolds for Stochastic Equations in Infinite Dimension. Journal of Functional Analysis 197, 398-432

[13] Gawarecki, L., Mandrekar, V. (2010): Stochastic Differential Equations in Infinite Dimensions with Applications to Stochastic Partial Differentail Equations (Probability and Its Applications). Springer

[14] Filipović, D., Teichmann, J. (2004): On the Geometry of the Term Structure of Interest Rates. Proceedings of The Royal Society A Mathematical Physical and Engineering Sciences 460, 129-167

[15] Hagan, P. S., Kumar, D., Lesniewski, A. S., Woodward, D. E. (2002): Managing Smile Risk. Wilmot 1, 84-108

[16] Kennedy, D. P. (1994): The Term structure of Interest Rates as A Gaussian Random Field. Mathematical Finance 4, 247-258

[17] Musiela, M. (1993): Stochastic PDEs and Term Structure Models. Journées internationales de finance IGR-AFFI. La Baule

[18] Pazy, A. (1992): Semigroups of Linear Operators and Applications to Partial Differential Equations (Applied Mathematical Sciences). Springer

[19] Potters, M., Bouchaud, J-P., Sagna, N., Cont, R., El Karoui, N. (1999): Phenomenology of The Interest Rate Curve. Applied Mathematical Finance 6, 209-232

[20] Zhang, X., Li, Y., Chen, P. (2016): On the Initial Value Problem of Stochastic Evolution Equations in Hilbert Spaces. Journal of Function Spaces 2016

UnIVERsity PARIS 1-SoRbonNe

Email address: rdouady@univ-paris1.fr
Stony Brook University

zeyu.cao@stonybrook.edu 